Nicholas, David B., Hedley, Darren,

Randolph, Jena K., Raymaker, Dora M., Robertson, Scott M. and Vincent, Jonathan ORCID: https://orcid.org/0000-0001-6278-4670 (2019) An Expert Discussion on Employment in Autism. Autism in Adulthood, 1 (3). pp. 162-169.

Downloaded from: http://ray.yorksj.ac.uk/id/eprint/4445/

The version presented here may differ from the published version or version of record. If you intend to cite from the work you are advised to consult the publisher's version: http://dx.doi.org/10.1089/aut.2019.29003.djn

Research at York St John $(\mathrm{RaY})$ is an institutional repository. It supports the principles of open access by making the research outputs of the University available in digital form. Copyright of the items stored in RaY reside with the authors and/or other copyright owners. Users may access full text items free of charge, and may download a copy for private study or non-commercial research. For further reuse terms, see licence terms governing individual outputs. Institutional Repository Policy Statement

\title{
RaY
}

Research at the University of York St John

For more information please contact RaY at ray@yorksj.ac.uk 


\section{An Expert Discussion on Employment in Autism}

Moderator: David Nicholas, PhD (1)

Participants:

Darren Hedley, PhD (2), Jena Randolph, PhD (3), Dora M. Raymaker, PhD (4), Scott Robertson, PhD (5), Jonathan Vincent, PhD (6)

(1) Faculty of Social Work, Central and Northern Alberta Region, University of Calgary, Edmonton, Canada

(2) Olga Tennison Autism Research Centre, School of Psychology \& Public Health, La Trobe University, Melbourne, Australia

(3) Thompson Center for Autism and Neurodevelopmental Disorders, University of Missouri, Columbia, USA

(4) School of Social Work, Regional Research Institute, Portland State University, Portland, USA

(5) Scott Michael Robertson Consulting, Washington, DC, USA

(6) School of Education, York St. John University, York, UK 
Introduction

The employment rate among autistic adults remains low. In the United States, over half of autistic youth were reported to have no engagement in employment or post-secondary education within two years after leaving high school. ${ }^{1}$ Only $14.3 \%$ of Canadian autistic adults (18-64 years) are reported to be employed. ${ }^{2}$ Approximately $28 \%$ of autistic adults in Australia report employment, ${ }^{3}$ and a survey of 2,000 autistic adults (or someone answering on their behalf) in the United Kingdom found only $32 \%$ in paid employment (either part-time or full-time). ${ }^{4}$ Pathways and processes associated with autistic adults' employment and career development are not well understood. ${ }^{5}$ Recent literature increasingly supports a range of considerations and resources to improve employment options and outcomes. ${ }^{6,7}$ Yet greater understanding is needed in ascertaining what employment means to autistic adults, and how to improve employment experiences and outcomes. The following is a transcript of a recent roundtable discussion on employment and autism, with edits for clarity and manuscript development.

Dr. David Nicholas: We have brought together five discussants who come from different disciplines and bring varying perspectives on this important topic of employment and autism. Two discussants are autistic themselves. Our goal in this roundtable is to amplify some key considerations and priorities in moving forward. Would each of you briefly introduce yourself?

Dr Darren Hedley: I am a Research Fellow at the Olga Tennison Autism Research Centre within the School of Psychology and Public Health at La Trobe University, Melbourne, Australia. My research has two main streams, the first addresses co-occurring mental health conditions in autism, and the second is focused on employment. We have been doing employment research for nearly 5 years, with this work funded by DXC Technology, the Australian Government Departments of Human Services and Defence. My background is in psychology, and I've been working with autistic individuals in various capacities, including intervention, clinically, and in a research capacity, for over 15 years. I think of my role as translating the experiences and voices of autistic individuals to reach different audiences (e.g., academics, policy makers).

Dr. Jena Randolph: I am an Assistant Professor and the Training and Education Division Director at the University of Missouri's Thompson Center for Autism and Neurodevelopmental Disorders. I have over 20 years of experience with individuals with autism throughout the lifespan across home, clinical, and school settings. Most recently, I am a co-developer of the Self-Determined Transition Readiness through Individual Vocational Experiences (STRIVE) program, which provides direct instruction, work experiences, and peer mentorship to enhance employment readiness for young adults with autism, and the co-developer of the Autism Friendly Business (AFB) program, which empowers businesses to support employment for individuals of all abilities.

Dr. Dora Raymaker: I'm a Research Assistant Professor at Portland State University's Regional Research Institute for Human Services. I'm a systems scientist, and I conduct applied social services research using a community-based participatory research approach in collaboration with the Autistic and other neurodivergent communities. I'm a co-founder and co-director of the Academic Autism Spectrum Partnership in Research and Education (AASPIRE), and a long-time neurodiversity and LGBTQ+ rights activist. My primary research focus is on improving skilled employment for autistic and other neurodivergent professionals, fueled in part by my own experiences as a service user. I'm also a science fiction author, which is less of a non-sequitur than it may sound. My stories, like my novel Hoshi and the Red City Circuit, frequently explore themes of disability and employment.

Dr. Scott Robertson: I am an autistic adult in Washington, DC. I have served as a co-founder and the Founding Vice President of the Autistic Self Advocacy Network (ASAN), as well as a Public Member of the Interagency Autism Coordinating Committee. I have also served as a Joseph P. Kennedy, Jr. Public Policy 
Fellow in the U.S. Senate Committee on Health, Education, Labor, and Pensions. During my career, I have led and supported initiatives to improve access to community living and employment for people with disabilities through other roles in government and the nonprofit world. I have also engaged as a collaborating researcher for occupational therapy and interdisciplinary research studies. My colleagues and I have conducted qualitative studies to examine the perspectives and experiences of autistic people on quality of life, employment, and related subjects. I feel passionate about this work because of my own challenges in securing work to fit my skills and talents.

Dr. Jonathan Vincent: I am a Senior Lecturer in the School of Education at York St. John University in the United Kingdom. My research focuses on the barriers and pathways for autistic students as they transition into, through, and from higher education. Most recently, I have been studying the employment ecosystem of autistic graduates to uncover their experiences of accessing work, as well as the attitudes and levels of knowledge among industry professionals. Where possible, my research is participatory and I am the Director of PRO Autism, which delivers training, co-facilitated by autistic experts-by-experience, to employers on how best to recruit and develop autistic talent.

\section{Dr. Nicholas: Thank you and let's begin. What do you see as priorities, goals or desired outcomes related to employment for people on the autism spectrum?}

Dr. Hedley: I will start by acknowledging the input of two of my colleagues, Dr. Simon Bury and Dr. Rebecca Flower-whose ideas I have incorporated into my responses.

First, employment needs to be meaningful, ${ }^{8}$ as well as stable and reliable. It should match the preferences of the individual, and the work environment should be respectful and inclusive. It needs to provide opportunities for inclusion, but also not force the individual to participate in, for example, extracurricular work events if they do not want to. They need to feel included and not separate from the non-autistic workforce.

How we get to this point is another matter. We need to be thinking about removing barriers and providing career pathways, not just jobs. We need to break down myths and stereotypes. We need to be strengths-based, while acknowledging challenges and support needs on an individual basis. And we need to ensure that we are not inappropriately applying values from non-autistic groups to the autistic population.

There are some points about the strength-based approach that Professor Stephen Shore talked about. ${ }^{9}$ He noted that there is a wide, varying skill set within the autistic population: "Although we may have incredible strengths in certain areas, it is also important to realize there are often significant challenges in need of support" (p.84). This aligns with the perspective that we have taken in our research, reflective of the message that we get from the autistic employees with whom we work. ${ }^{8,10}$ It is really important to take an individualistic approach and look at what each person offers. We really need to consider individual preferences about what people actually want to do. It is also incredibly important to identify and provide whatever supports are required to ensure that people can achieve this potential.

Dr. Randolph: Many adults with autism are either unemployed or underemployed. Our biggest goal is to increase inclusion opportunities for them. We have come a few steps forward in the U.S. with the passage of the Workforce Innovation and Opportunity Act (WIOA), which promotes opportunities within inclusive competitive employment settings. But we have a long way to go to increase wages, hours worked, and opportunities in general. As an offshoot of this, and as a major goal, we need to look at how we support employers and communities to truly be inclusive because there are a lot of misconceptions, and some people seem to not yet fully embrace inclusive employment. I think we can break down those barriers. 
Dr. Raymaker: I have always been interested in how good employment outcomes in a lot of the service systems may be somewhat different from what autistic people themselves want. I recently did a large qualitative study where I asked autistic professionals, "What does a successful employment outcome look like to you?". I could summarize the whole thing as, "We want what everybody else wants." Some of the things that people named were professional growth, work-life balance, financial independence, a sense of community from their workplace, feeling valued, doing meaningful work, and having an accepting neurodiversity, or autistic disability-friendly, work culture-a place that they could go where they felt belonging.

Another thing that I learned from the people whom I spoke to is that this does not always necessarily look the way it does for nondisabled professionals. We may need to work fewer hours, skip certain office activities, or have hard skills privileged over soft skills.

And so, while inclusion and paying us all the same wage and those types of things are really important, we do not necessarily always want these elements to look or be the same. But this must be balanced with the idea that we do want the same outcomes as everyone else.

I would also add that employment is connected to a lot of good health and wellness outcomes. So, people who are employed are more likely to have better mental and physical health. But to attain those benefits, you also need a work-life balance where your work is not contributing to your mental or physical health problems. I would like to see more of a holistic approach in looking at employment and seeing how it connects to the other parts of a person's life and well-being.

Dr. Robertson: The emphasis on tapping strengths is tremendously important. We must strive to align the workplace situation and environment to fit the person rather than try to change the person to fit the environment. I also see a critical need to expand the research literature on improving employment opportunities for autistic youth and adults. We need more studies that examine perspectives of autistic people ourselves.

A desired outcome is to explore how to achieve greater access to meaningful careers and employment opportunities that can effectively harness our talents and skills. Speaking from my personal experience as an autistic person, I have the good fortune of building and enhancing a career that fits my own focused interests. I strongly agree with the focus on careers - not just jobs. Many autistic people regularly encounter numerous barriers to attaining supports and accommodations to grow careers.

Thus, I believe we need to encourage a paradigm shift toward supporting talents and skills. Equally, we need to ensure that employment supports for autistic people can align with employers' business needs for valued talents and skills. The literature has begun to improve in this area as we are seeing more research studies focusing on employer engagement and support systems to facilitate work skills.

An equally important goal is to examine how employment opportunities drive economic well-being for autistic people. Despite limited research on the economic well-being of autistic people, first-hand narratives give us strong reason to believe that many autistic youth and adults face substantial socioeconomic adversity; this represents the reality for the cross-disability community. Some studies from the United Kingdom indicate that autistic people may have a higher rate of homelessness than non-autistic people. Thus, we must consider socioeconomic status and the overall quality of life of autistic people when conducting employment research and innovating best and promising practices to increase employment opportunities. We must also ensure that employment studies include diverse autistic people; autism research has not historically prioritized this focus.

Dr. Vincent: When I was thinking about this question, "What do I see as the goals or desired outcomes?" I thought it is not for me to identify what the goals are; it is for the individuals themselves. Dr. Julie 
Lounds Taylor wrote a paper in which she asked the question, "When Is a good outcome actually good?". ${ }^{11}$ That is about going back and concluding, 'it is about people', and how do we make sure that our goals and outcomes are what are right for the person in particular. In terms of goals and outcomes, it is really for autistic people to identify their goals.

I am interested in the Capability Approach developed by Amartya Sen, an Indian economist, ${ }^{12}$ who looked at well-being as the ultimate goal. Sen would say, "The best thing is whatever leads to a flourishing life." What that ultimately means is that it's about having choice, having capabilities to choose what your life is, how you lead your life, and what you can be and do. Rather than having a predefined set of goals, it is diverse, which I think also speaks to the autistic population more generally, which is diverse. Autistic people want to do a whole range of different jobs. My research has shown that not all autistic students want to work full-time or in traditional employment. But they all want to be in control and have freedom and opportunities to make choices about their lives. So, I think that the goal should be to shift the discourse toward asking autistic people about what they see as their goals.

\section{Dr. Nicholas: What are both the greatest barriers and facilitators toward achieving the aims that you have conveyed?}

Dr. Hedley: Better physical and mental health outcomes can be associated with employment. However, a stressful work environment may actually contribute to poor outcomes. For example, a poor fit with the environment or an environment that does not accommodate needs or support the autistic individual could contribute to stress and anxiety-related problems.

Another barrier reflects a point that Dr. Robertson raised in describing the need for a paradigm shift. The traditional recruitment process-one where you have a job description, and the person selected for that job will be the best fit to that job description-does not help autistic individuals get work. We need a paradigm shift; we need to turn that process on its head and identify the strengths of the individual, their abilities, and what contribution they might make to an organization or work environment-rather than the other way around.

When we talk to participants in our program, they describe benefits from being involved. The program that we work with provides supports for individuals, and also targets autistic individuals for employment. They convey that the benefits of the program are getting a foot in the door and having an opportunity, and they describe how important this is to being competitive for open employment later. The flip side of this is the difficulty that autistic individuals have getting a foot in the door. Again, this has a lot to do with traditional recruitment practices and human resource processes. ${ }^{13}$ The job interview, we have found, is a massive barrier to accessing employment, ${ }^{8}$ particularly when autistic individuals are competing against non-autistic individuals in an interview that is reliant on making a good first impression and social interaction between the interviewer and the interviewee.

Other barriers include myths and stereotypes surrounding autism. ${ }^{10}$ In our research, participants say that when they go into a workforce, people are expecting a Rain Man or a computer genius or a hacker, and there is not enough awareness of what autism actually is - at least that first impression. After time, these barriers break down. When we talk to coworkers, they say, "Oh, actually, these people are a lot like us. We did not realize there were so many similarities."

In terms of facilitators, we have found that autism awareness in a workplace is important. ${ }^{8}$ Coworkers report benefits from some understanding about autism in working with their colleagues on the spectrum, including understanding why certain behaviors or difficulties may occur, and how these might be related to autistic traits. 
Flexibility is really important-flexibility in work hours, for example. Participants in our program report the importance of receiving positive feedback and predictability in the workplace. One of the most important things that we have identified in our research is having someone to whom the autistic person or the work colleague can go to resolve issues.

If work problems are related to soft skills, most seem to be resolved by having a person who understands autism, serving as a mediator between the autistic individual and wherever the problem is occurring in the workplace. Champions and advocates in the workplace are important to success.

Dr. Randolph: We still have limited access to the varied services and opportunities that could help individuals to meet their goals. Barriers that feed into that include under-utilization of braided funding streams to make more opportunities available. Adults with autism from rural areas and with fewer socioeconomic resources have even greater barriers to access.

Employer readiness is a huge key. There are many barriers in the recruitment and interviewing process. Employers need to understand how to be more flexible, not just in those initial stages, but also in job modification. Are we looking at carving positions that feed into individual strengths? Are we modifying on the job the type and amount of work? Can we better utilize peer mentors and peer mentorship programs? There are a lot of universal and natural supports within employment sites that could readily and easily help facilitate better employment outcomes.

For rural areas specifically, technology could be used to provide better access to services. We need more employer training and incentives for businesses to participate in such training, including opportunities for businesses to access consultation when needed.

Dr. Raymaker: The human resources issues are huge. A huge facilitator of success was reported to be nontraditional, odd pathways that you would never expect like, "I was the first person who ever used this type of equipment. So, I became the only expert in it." An added layer is workplace trusted alliesnot just workplace supervisors and coworkers, but also job coaches and employment support professionals. Having an actual friendship relationship or someone who genuinely cares is key.

A huge barrier to success in our studies consisted of pressures to mask autism or other marginalized identities - having to hide who you are, not being able to get accommodations, and working in an environment where you did not feel comfortable disclosing or asking for accommodations. The combined strain of that led to a lot of bad situations. In contrast, job cultures that accommodated and led autistic people to be themselves, including safe disclosure, as well as just being able to act autistic at work, were huge facilitators of success.

Another big barrier is a history of 'failure' in getting and keeping jobs, and also abuse, bullying, and trauma. Trauma that has been picked up usually early on, transfers into work settings where you then expect the boss to be mean to you, are afraid to talk to anyone, and have to say "yes" to everybody because you are afraid that you are going to get hurt and lose your job if you say "no" to something you cannot do. Those sorts of historical traumas and failures build up. So, in talking to people who were fresh out of their training and looking for a job, they were generally pretty optimistic and could shrug off some failure, and they felt good about their career. But when talking to people who for 50 years have been having successive failures and abuse, their ability to really be successful in the workplace is impeded by all of that trauma and fear that gets carried through, and then employers often do not understand it, and just think, "Oh well, they are being difficult. We do not want this person here." So, early successes are a facilitator - if there can be some early fast wins.

Dr. Robertson: Autistic people often experience trauma and adversity from bullying and other challenging life circumstances throughout childhood and adult life. Many of us may develop negative 
perceptions from these experiences that can lead to frequent worrying about doing things wrong. We are also often told that we are broken or not competent. These negative experiences can collectively hamper a person's ability to seek employment, which means we need greater supports, including in the workplace, to cultivate positive life experiences.

I will emphasize that our discussion has Universal Design implications; for instance, mentoring, training, and customized work environments benefit all workers. We have also discussed the work-environment fit, which can present a double-edged sword. When work-environment fit is poor and the focus is on changing the person, this situation does not support equal access for autistic people. We often adjust work opportunities to bring in talented workers. Yet, when someone has a disability, work situations often do not incorporate these same kinds of workplace adjustments that equally benefit companies.

I also find it important to consider the context for autistic job seekers. Many individuals may have a work history that looks significantly atypical; they may have worked for a few or several months at a job and then moved on, and then again move to other jobs, and so forth. Many autistic people are not receiving the supports and accommodations needed to be successful and, thus, they often must change jobs frequently. This work history can hinder attaining a future good job that does align with their talents and skills; employers may have concerns about whether a job candidate is going to stay at the job. Employers should seek to understand this context, and try to develop a perspective from the job seeker on how accommodations and supports can ensure that the talents and skills of the individual will benefit the company.

The autism research literature also tends to center on deficits of autistic people while underemphasizing our assets and core strengths. Employment studies and initiatives on autism should focus more on elements of positive psychology, such as resilience. Likewise, we should value how selfdetermination and self-advocacy can facilitate employment.

Dr. Vincent: Fundamentally, I think the biggest barrier is a lack of knowledge, but it is also misinformation-myths and expectations that are unfair or untrue. That feeds into recruitment processes, job advertisements which are written in particular ways, interview processes, and psychometric testing-which are set up with little if any consideration for neurodiversity. All of these come from a lack of understanding.

From my research, a barrier is a lack of trust in employers among autistic students and graduates. And I think that relates to a sense of failure, and particularly fear and a lack of trust in terms of how employers will respond to disclosure. Will they respond positively or put the application to one side? Will they perhaps offer an interview but if so, it feels like it is out of sympathy or charity? So, not feeling confident to disclose definitely seems like one of the biggest barriers.

Other people on the spectrum do not necessarily see their diagnosis as a disability. Many people with whom I have spoken say, "I don't tick the box to say I am disabled, because I don't see it as a disability." A kind of catch-22 emerges as employers say, "If we don't know that someone is autistic, we cannot make adjustments, and then it is not our fault if we don't employ them." So, they are saying, "We want you to disclose," but autistic people do not feel confident in doing that.

From my perspective related to autistic students, but probably for many people, a helpful facilitator can be working with individuals to map out a plan towards achieving personal goals, and not waiting to do so until the end of university.

\section{Dr. Nicholas: Are there key elements to improve employment outcomes?}

Dr. Hedley: I want to pick up on something that Dr. Vincent mentioned about disclosure. Feedback that we got from people in our research is that there may be some middle ground related to considerations 
like who you disclose to. For example, is it necessary to disclose to everyone in a workplace? It might be helpful to disclose to a supervisor or manager, but not more broadly. It may depend on the knowledge that a person has about autism. And is it safe? When do you disclose-in the interview, before you get the job, or after you have got the job? And asking the question "why?" seems important. This might relate to the job-person fit. Is it necessary to get supports to do the job? So, in terms of disclosure, there needs to be more discussion around that.

There is need for appropriate support in the workplace, including mental health support. ${ }^{14}$ Mental health and well-being is so important to sustainability, and a lack of mental health is possibly one of the biggest barriers to employment.

Internships and placements are an excellent way of increasing participation in the labor force through helping individuals get experience, and exposing industry to individuals.

There seems to be a cliff at the end of higher education relative to employment. People finish their degrees or certificates, but then are not able to transition to employment. There needs to be something in place in post-secondary educational institutions to assist with that transition period. A good example is providing internship opportunities and connecting industry to the higher education system. Industry needs to be informed about autism, including supports that might be required to create sustainable employment.

Alternative or entrepreneurial-type ventures need to be supported. This might include small or family businesses that could support one's autistic talents, for example, artistic talents. ${ }^{15}$ There is often a focus on large organizations and industry, but there are many small to medium-sized businesses that need to be tapped into; accordingly, the big organizations are only part of the solution.

There is also a need for greater focus on policy change. This might include guidelines and recommendations at the government level which are aimed at increasing diversity in work settings. Needed advances include training, incentives, and changes to HR policy and recruitment strategiespossibly even workplace quotas.

Dr. Randolph: I want to focus on earlier years-long before employment is on the table (i.e., pretransition years). I think that the approach is twofold. It is not only how we empower individuals at a young age to reach their goals, but also how we prepare the community and employers to not only accept, but also embrace, the benefits of a neurodiverse workforce. We need schools to embrace a transition-focused mindset earlier, emphasizing individual strengths and personal goals, as well as focusing across the spectrum of ability and teaching skills for independence. In terms of challenges on the job, it is never the hard skills that we hear about; it is always the soft skills, such as executive functioning or the social nuances of navigating the work environment.

Employer engagement is necessary, including multi-faceted employer training. It starts with the benefits of employing neurodiverse individuals, and emphasizes the use of natural and universal supports, such as mentorship to foster a fully inclusive environment for everyone to succeed.

Dr. Raymaker: I particularly appreciate that people have been talking about ways to improve employment that do not place additional burden on the autistic person. That is something that is really important to me and to the community, and I am glad that we are at a stage where we are talking more in those terms. I think that what is required is a systems-wide solution. I do not think you can target any one piece of what we are talking about without looking at the whole, even things that are somewhat outside of the employment system, such as the supports that somebody is receiving. I remember talking to someone who could not get a job because they lived in a very rural community, but they could not 
leave the rural community because their disability supports existed in that community. If they were to go to an urban center, they would lose their ability to make life happen.

So, I think that my answer to what is required to improve outcomes is a greater amount of systemsthinking about how all of these components interact-where leverage might be found for different sorts of scenarios within that broader system.

People have mentioned policies and training. I also think we must consider cultural and historical context, which is part of what Dr. Robertson is getting at with the notion of a paradigm shift. I think that all of these things have to work together as a unit in order to really improve employment as a whole.

Dr. Robertson: Supporting disclosure is critical to improving outcomes since autistic people may have concerns about how to disclose in the workplace. Autistic employees and job seekers need to be met where we are when ensuring that we can disclose in ways that address our concerns. Sometimes that may mean selective disclosure. Some autistic people may not necessarily always want to discuss their autism. Others may want to disclose to their supervisors, but not tell coworkers. I find it critical to support flexibility for disclosure. Selective disclosure may mean that autistic workers can state, for instance, "Fluorescent lights bother me," or, "I just need a quieter space."

Supporting disclosure ensures that autistic employees can access needed workplace accommodations, adjustments, and supports. Many of these accommodations and supports that we have been discussing do not have a high associated cost; they just require thinking differently.

Improving outcomes requires adjusting attitudes, and emphasizing approaches that can support better outcomes. Autism studies often lack depth in identifying specific strategies for improving employment outcomes. It is also tremendously important that researchers and practitioners seek to learn more from autistic people, including strategies, approaches, technology aids, and other supports.

This situation has begun to improve in the last 5-10 years. However, historically, researchers have often not tapped the perspectives of autistic people. Autistic people like me who are researchers have frequently lacked supports to ensure we are integrated and fully included in the research process. I think that researchers have increasingly heeded this concern, including when they are conducting communitybased research. Partnerships, including those with initiatives and organizations run by autistic people and allies, can critically drive improved outcomes; we can learn first-hand from successes, achievements, and accomplishments of autistic people, including which supports, services, and accommodations facilitate access to jobs matching our interests, skills, and talents.

At the same time, research and initiatives should explore key challenges and barriers because it is equally important to examine what went wrong, and what we should learn not to do. We must also remember that autistic people need the flexibility to shift our careers as our interests and experiences change; we should support self-determination for autistic people to make job shifts and change careers as for non-autistic people.

Dr. Vincent: In terms of what is required to improve employment outcomes, a word that kind of sums it up for me is dialogue. It comes from an autistic colleague of mine and former company owner himself who said it simply comes down to having a conversation at industry or sector levels, as well as at a more granular workplace level. I think that notion of having a dialogue is central.

An important facilitator of success is having people-allies in a workplace-who typically are not the manager but can advocate and care. There is also something around dialogue between employers. For improved outcomes, an important aim is changing employers' and industry professionals' perceptions. That is very hard to do from the outside. I think the best way that this will happen is for people within 
companies who have seen the success of autistic people, to move beyond and advance this notion not as merely socially good but rather as good for companies.

The last thing that is important is the idea of the ecosystem. Quite often within research, employment is seen as just something which an individual does, which is never the case. There are so many other factors; it is multifactorial. As Dr. Robertson mentioned, the socioeconomic context is important. And the political context is important, as is the support of employment providers. There are parents, carers, and schools and universities - all of those elements work together. The more we can understand the ways in which people are looking for employment and being successful in it, as well as being deprived of employment-and how that happens within a complex ecosystem, the better we will get at finding solutions.

\section{Dr. Nicholas: How should we ensure that employment is proactively addressed across the diversity of autism, including demographic diversity?}

Dr. Raymaker: We have talked about disclosure as being about support and getting accommodations, but I believe that it is more than that. Disclosure is also about being able to take off the mask. Even if you do not need accommodations, being able to fully be yourself and feel accepted as yourself, is very important.

There is a lot more to a person than just their disability, and autism disability programs are really focused on autism. People have intersectional identities, and they may be multiply disabled. They may be multiply marginalized. And especially when you are talking about employment, these other identities have a huge impact. I have talked to people for whom their racial identity was actually a much larger barrier to employment than their autism. Some would choose what to disclose based on what they can or cannot hide.

People making these types of decisions and negotiations between their identities know that each of those identities is going to cause them risk-risk of not getting or losing their job, or of being bullied. So, I think that the idea of diversity is one that has been insufficiently looked at and is absolutely critical to bring into a broader context.

We need to find better ways to connect and capacitate existing support and service systems to recognize and convey, "We are going to work on getting you a job that takes into consideration that you are on the spectrum, but do you want to talk about the fact that you are trans? Do you worry about how people will react to that in the workplace? Can we help?"

Dr. Hedley: There are multiple diversities we need to consider-gender is one. Most of our hiring programs seem to be hiring males. I think this is an area for future research.

It has been good to see that large multinational organizations are working together to address and increase diversity in the workplace. There is a lot of work that needs to be done around how we do this, but it is good to see that there is a dialogue.

My final point is on demographic diversity. Dr. Daniel Leong, an autistic advocate from Malaysia, has been traveling to promote autism awareness for both industry and universities. He has set up a group of autistic individuals for autistic individuals, ranging from those who are highly verbal to those who are more limited with regard to verbal abilities. Group members advocate for one another. They are doing an amazing grassroots-level job of raising awareness about the capabilities and potential contributions of autistic individuals to the workplace, as well as the barriers preventing access. Change requires work from individuals themselves as well as from families, interested groups, and organizations at different levels. We know that individuals can be competitive and productive at work, but need to be given the opportunity to tell their story, and they need employees and others to listen to that story. 
We need to make sure that we are not concentrating only on Western countries. It is important to give attention to success stories and the amazing work that is occurring globally, as well as to the work that needs to be done.

Dr. Randolph: We need more research exploring technology to serve rural communities. I would also like to see technology utilized for employment site readiness, and where appropriate, more individualized and ongoing support.

We need to be better at sharing success stories across the spectrum, including those of individuals with autism and intellectual disabilities. I think that this could help break down some of the stereotypes that we see perpetuated through the media.

Dr. Robertson: We are becoming better at seeking to include people with intellectual disability and other co-occurring disabilities in autism research on employment. We also are making some strides in the inclusion of individuals representing diversity of race/ethnicity, socioeconomic status, gender, gender identity, sexual orientation, and age in research and innovation of practice. Autism research has reflected a bias toward autistic people who are male, white, from higher socioeconomic status, and not users of augmentative and alternative communication (AAC). We should effect change by re-shaping this paradigm and shifting perspectives and norms in research and professional communities.

Many autistic people use AAC part-time or all the time, and employment research and initiatives should represent this range of communication experience. Community-based research, partnerships with organizations run by and for autistic people, and connection-building with online communities of diverse autistic people can support efforts to include diverse autistic people.

Dr. Vincent: For too long, research has focused on one particular demographic within the autistic population. It has been positive to see a shift more recently in terms of the intersections between autism and gender. But I think we still have a long way to go in terms of recognizing that there is a wide range of identity intersections-multiple factors which can make employment much more difficult. This is definitely an area in which we need to do better, particularly for individuals with higher support needs whose voices are rarely heard.

\section{Dr. Nicholas: If you had to select only one or two priorities to improve employment outcomes, what would they be, and how would you move them forward?}

Dr. Raymaker: My priority right now is workplace culture shift. And as far as how am I going to push it forward, I just received a grant to look at the general employment organizational psychology literature and solutions because there actually is much known about workplace culture shifts with other populations. I want to look outside of autism at the way that some of these problems may have been understood in other contexts and populations.

Dr. Vincent: A priority is to develop understanding in creating genuine, productive dialogue that needs to take place in workplaces. And I think that is only going to come about when autistic people and their allies are enabled to be involved in training, and to advocate for themselves and others about the potential that they have-the autistic strengths that they can bring to the workplace, with the right kind of adjustments. To this end, a priority is more knowledge and understanding generated around these considerations. Out of that, I think, comes better recruitment processes and strategies that enable people to be successful throughout their careers.

Dr. Hedley: We definitely need to break through some of the myths and stereotypes surrounding autism, and spread evidence-based knowledge about autism and what it is like to be autistic, how diverse autism is, and the contribution that autistic individuals can and do make to society. My second priority is around the need for government-level policy that recognizes and rewards employment 
diversity compliance, and enables organizations to increase diversity targets. We are finding that organizations are very motivated, but they lack the how (i.e., knowledge for implementation), and they are risk-averse. We need education for employers, including large multinational organizations, small or medium enterprises, and government organizations. They need to be provided with knowledge and tools to achieve these goals. Most importantly, diversity goals must be genuine and not tokenistic.

Dr. Randolph: My biggest priority is increasing employer knowledge and readiness. This goes beyond broad-based training to businesses and people in managerial positions, and includes giving businesses access to ongoing support and individualized consultation when needed.

Dr. Robertson: My first priority is better data on concrete strategies and approaches that are working. We also need more communities of practice and outlets for sharing resources, information, and data. My second priority is to ensure that autistic people can serve as partners and collaborators in research and other initiatives. For instance, autistic researchers who study employment can connect with practitioners and other researchers. Community-based participatory research supports this goal. My final priority is diversity of background and experience. We need to drive the inclusion of autistic individuals with broad support needs, varied talents and skills, and diverse backgrounds such as race/ethnicity, gender, sexual orientation, gender identity, and socioeconomic status.

Dr. Nicholas: Thank you to our panelists for these rich insights and considerations. As I reflect on your commentary, it seems that some headway has been made in recent years, but there is much yet to be done. I hope that that this important conversation moves forward, along with proactive action in advancing employment opportunity for autistic adults.

\section{Acknowledgements:}

We would like to thank the Autism in Adulthood editorial board members for selecting this topic. We are especially grateful to board members Marc Fabri, Finn Gardiner, Steven Kapp, Anne V. Kirby, Helen McConachie, Kim Musheno, Christina Nicolaidis, Stephen Shore, Nidhi Singhal, and Teresia Waisman for their help identifying potential discussants, preparing Roundtable questions and commenting on the transcript. 


\section{References}

1. Shattuck PT, Narendorf SC, Cooper B, Sterzing PR, Wagner M, Taylor JL. Postsecondary education and employment among youth with an autism spectrum disorder. Pediatrics. 2012;129(6): 1042-1049. https://doi.org/10.1542/peds.2011-2864

2. Zwicker JD, Zaresani A, Emery JCH. Describing unmet needs among adults with a developmental disability: An examination of the 2012 Canadian Survey on Disability. Res Dev Disabil. 2017;65:1-11. https://doi.org/10.1016/ j.ridd.2017.04.003.

3. Australia Bureau of Statistics. Disability, Ageing and Carers, Australia: Summary of Findings, 2015. https://www.abs.gov.au/ausstats/abs@.nsf/Latestproducts/4430.0Main\%20Features752015. Published 2017. Accessed July 17, 2019.

4. National Autistic Society. Government Must Tackle the Autism Employment Gap. https://www.autism.org.uk/get-involved/media-centre/news/2016-10-27-employment-gap.aspx. Published 2016. Accessed July 17, 2019.

5. Taylor JL, McPheeters ML, Sathe NA, Dove D, Veenstra-VanderWeele J, Warren Z. A systematic review of vocational interventions for young adults with Autism Spectrum Disorders. Pediatrics. 2012;130(3): 531-538. https://doi.org/10.1542/peds.2012-0682

6. Nicholas DB, Mitchell W, Dudley C, Zulla R, Clarke M. An ecosystem approach to employment and autism spectrum disorder. J Autism Dev Disord. 2018;48(1): 264-275. https://doi.org/10.1007/s10803017-3351-6

7. Hedley D, Uljarević M, Cameron L, Halder S, Richdale A, Dissanayake C. Employment programmes and interventions targeting adults with autism spectrum disorder: A systematic review of the literature. Autism. 2017;21(8): 929-941. https://doi.org/10.1177/1362361316661855

8. Hedley D, Cai R, Uljarević M, Wilmot M, Spoor J, Richdale A, Dissanayake C. Transition to work: Perspectives from the autism spectrum. Autism. 2018;22: 528-541.

https://doi.org/10.1177/1362361316687697.

9. Urbanowicz A, Nicolaidas C, de Houting J, Shore SM, Gaudion K, Girdler S, Savarese RJ. An expert discussion on strengths-based approaches in autism. Autism Adulthood. 2019;1(2): 82-89.

10. Bury SM, Hedley D, Uljarević M, Dissanayake C, Gal E. If you've employed one person with autism...: An individual difference approach to the autism advantage at work. Autism. 2019;23(6): 1607-1608. https://doi.org/10.1177/1362361318794937

11. Taylor JL. When is a good outcome actually good? Autism. 2017;21(8): 918-919. https://doi.org/10.1177/1362361317728821

12. Sen AK. The Idea of Justice. Cambridge, MA: Harvard University Press; 2009.

13. Krzeminska A, Bruyere S, Austin R, Hedley D. Advantages and challenges of neurodiversity employment in organizations. Journal of Management and Organization. In press. 
14. Hedley D, Uljarević M, Bury SM, Dissanayake C. Predictors of mental health and well-being in employed adults with Autism Spectrum Disorder at 12-month follow-up. Autism Res. 2019;12: 482-494. https://doi.org/10.1002/aur.2064

15. Hedley D, Uljarević M, Hedley DFE. Employment and living with autism: Personal, social and economic impact. In: Halder S, Assaf LC, eds. Inclusion, Disability and Culture: An Ethnographic Perspective Traversing Abilities and Challenges. New York: Springer; 2017;295-311. 